\title{
A pilot study on morphology and the mechanism involved in linearly patterned programmed cell necrosis in melanoma
}

\author{
CHUNRONG HAN ${ }^{1,2}$, BAOCUN SUN ${ }^{1,3}$, WEI WANG ${ }^{1,4}$, WENJUAN CAI ${ }^{1,4}$, \\ DAN LOU ${ }^{3}$, YAN SUN ${ }^{1}$ and XIULAN ZHAO 3 \\ ${ }^{1}$ Department of Pathology, Tianjin Cancer Hospital and Institute, Tianjin Medical University, Tianjin; \\ ${ }^{2}$ Department of Pathology, Yangzhou Hongquan Hospital, Jiangsu; ${ }^{3}$ Department of Pathology, \\ Tianjin Medical University, Tianjin; ${ }^{4}$ Tianjin First Center Hospital, Tianjin, P.R. China
}

Received March 3, 2010; Accepted July 8, 2010

DOI: 10.3892/ol_00000144

\begin{abstract}
Accumulating data provide evidence that autophagy contributes to programmed cell death (PCD) under certain circumstances. Immunohistochemistry and real-time PCR were performed to investigate the correlation the expression of autophagy-related proteins LC3 and Atg4B and linearly patterned programmed cell necrosis (LPPCN) in melanoma. LPPCN was recently reported to be a special PCD, which is similar to neither apoptosis nor conventional necrosis commonly observed in tumoral tissues. The mechanism involved in LPPCN remains unclear. Our data showed that the expression of LC3 and Atg4B in the LPPCN-positive group was significantly higher than that in the LPPCN-negative group regarding protein and mRNA levels $(\mathrm{p}<0.05)$. Based on morphological observation, immunohistochemistry and real-time PCR experiments in this study, it was concluded that autophagy may play a crucial role in the process of $\mathrm{LPP}+\mathrm{CN}$ in melanoma. This study provides novel insights into the mechanism that regulates LPPCN in vivo during tumor development. We speculated that LPPCN may be an early stage event in tumoral neovascularization under hypoxic-microenvironmental conditions. Accordingly, LPPCN can be considered a novel target in the process of antiangiogenesis treatment, which can be expected to obtain a better clinical outcome in the future.
\end{abstract}

\section{Introduction}

Melanoma is characterized by rapid growth, mortality, unpredictable behaviour and resistance to chemotherapy, and surgery is currenlty considered to be the only effective therapy (1). The American Cancer Society estimated that melanoma accounted for 8,420 deaths in 2008 (2). Despite numerous

Correspondence to: Dr Baocun Sun, Department of Pathology, Tianjin Cancer Hospital, Tianjin Medical University, Tianjin 300060, P.R. China

E-mail: baocunsun@gmail.com

Key words: autophagy, linearly patterned programmed cell necrosis, melanoma years of continuous research, the histological characteristics and related mechanisms of melanoma remain unclear. Linearly patterned programmed cell necrosis (LPPCN), which was previously neglected by pathologists, was recently reported by Zhang et al (3) as being a special programmed cell death (PCD) in highly aggressive melanoma. LPPCN is morphologically characterized by loss of cell-cell adhesion, concentrated cytoplasm and the cellular nucleus being stained darker than that of the environmental cells in hematoxylin and eosin (H\&E)-stained sections. More importantly, cells that underwent LPPCN were distributed in patterns of lines and/or networks among the environmental tumor cells. LPPCN is similar to neither apoptosis nor conventional necrosis commonly observed in most tumoral tissues.

The mechanism involved in apoptosis is genetically regulated cell death and is a type of physiologically active death. However, a cell dying by conventional necrosis is regarded as a victim of extrinsic events beyond its control, meaning that conventional necrosis is a pathologic death and a passive process. Apoptosis is morphologically characterized by karyolysis, pyknosis (deep staining of nuclear mass), karyorrhexis and the formation of condensed cell bodies (apoptotic bodies) (4). This ordered morphology depends on the ability of the dying cell to engage in ATP-dependent processes of self-degradation. By contrast, necrosis can be defined morphologically by electron-lucent cytoplasm, swelling of cellular organelles and loss of plasma membrane integrity (5). Yuan et al (6) and Zong et al (7) described programmed necrosis as a novel cell death phenomenon in ischemic brain injury animal models. Morphologically, programmed necrosis exhibits characteristics that are different from the apoptotic bodies and DNA fragmentation typical of apoptosis, and different from the morphological features described above in conventional necrosis. Furthermore, apoptotic cells distribute randomly, and cells dying to conventional necrosis distribute in clusters and inflammatory cells occur in surroundings of necrosis in H\&E-stained sections, whereas cells that underwent LPPCN formed a line and/or network pattern. Thus, it appears that neither the morphological change nor the mechanism involved in LPPCN is similar to apoptosis or conventional necrosis.

The mechanism of this special cell death involved in LPPCN should be elucidated. Zhang et al (3) suggested that 
LPPCN is similar to mitochondria-dependent apoptosissignaling. Additionally, Endo G, which is an essential member of the mitochondria-dependent apoptosis signaling pathway, may play a role in the process of LPPCN. Recent technological advances have helped define the function and mechanism of programmed necrosis. Accumulating evidence has confirmed that autophagy plays an important role in cell survival and disintegration (8). Autophagy has also been suggested as a possible mechanism for non-apoptotic and/or non-necrosis cell death, despite evidence from a number of species that it is a survival strategy in times of stress (9). In autophagy, a doublemembrane structure known as an autophagosome, sequesters a section of cytoplasm and fuses with the lysosome/vacuole to deliver its contents into the lysosomal/vacuolar lumen. The encoding microtubule-associated protein light chain 3 (LC3), is an important autophagy-related protein with a mammalian ortholog of Atg8 in yeast. Atg4B is a critical regulator of LC3 (10). LC3 is localized to the autophagosomal membrane, and is widely used as a key molecule to monitor autophagosome formation and autophagy activity in mammalian systems. PCD has captured the attention of various cancer investigators. Numerous studies on the role of autophagy in PCD type II or non-apoptotic death are available (12-15). However, the role of autophagy in oncogenesis and progress remains unclear, and mechanisms that induce autophagy and regulate its outcome in human cancers have yet to be elucidated.

In this study, LPPCN was examined via morphological and experimental evidence. Moreover, the correlations between the expression of LC3 and Atg4B and LPPCN were investigated in order to identify the role of the autophagy-related proteins in the process of LPPCN, as well as to determine the mechanism and the clinicopathologic significance of LPPCN in melanoma.

\section{Materials and methods}

Patients. This study utilized 70 primary tumor specimens of human melanoma obtained from patients who consecutively underwent surgical resection in the Tianjin Medical University Cancer Institute and Hospital in China between January 1999 and October 2005. The patients comprised 27 women and 43 men with ages ranging from 28-81 (mean 54.96; SD 12.60). During the final follow-up, 12 patients had survived and 58 had succumbed within 113.5 months, with the median survival time being 29.5 months.

Clinical and histopathological evaluation. The tissue slides of 70 melanoma patients were reviewed using the World Health Organization Classification of Tumours Pathology and Genetics of Skin Tumours (11). Tissue sections of $5 \mu \mathrm{m}$ were cut from the formalin-fixed, paraffin-embedded representative tissue blocks of melanoma and were stained with H\&E. The quantities and distribution of LPPCN, and other characteristics involved in LPPCN, were evaluated in all 70 sections. The number of positive cells per $\mathrm{x} 200$ field was then assessed. The percentage of cells that underwent LPPCN was rated as: cases with $<1 \%$ positive cells were rated as zero, $1-25 \%$ positive cells as 1 point, $26-50 \%$ positive cells as 2 points and $>51 \%$ positive cells as 3 points. Clinicopathological parameters were obtained from patient medical records and files kept at the Department of Pathology, and included information on gender, age, tumor location, survival status, survival time, lymph node status, distant metastasis, AJCC stage (i.e., based on the American Joint Committee On Cancer Staging System), tumor thickness, coventional necrosis, histological subtypes, melanin, cellular phenotype, tumor-infiltrating lymphocytes and nucleolus.

Immunohistochemical staining. Serial $4 \mu \mathrm{m}$ sections cut from formalin-fixed, paraffin-embedded tumor tissue for immunohistochemical analysis were deparaffinized and hydrated through a series of xylenes and alcohols prior to immunohistochemical staining (using the alkaline phosphatase-streptavidin method) for $\mathrm{Ki}-67, \mathrm{HIF}-1 \alpha, \mathrm{LC} 3$ and Atg4B. The sections were rehydrated in phosphate-buffered saline (PBS), and antigen retrieval was performed using microwave heating for $5 \mathrm{~min}$ in 10 melanoma citrate buffer solution $(\mathrm{pH} \mathrm{6.0)}$ for survivin. The sections were incubated with monoclonal mouse anti-HIF-1 $\alpha$ (clone Sc-53546; Santa Cruz Biotechnology, dilution of 1:50), anti-Ki67 (ready-to-use; Zhongshan, Beijing, China), rabbit polyclonal anti-LC3 (Novus Biologicals, USA, dilution of 1:50), and rabbit polyclonal anti-Atg4B (Abzoom, USA, dilution of 1:50). Following incubation, the sections were rinsed with PBS and incubated with biotinylated goat anti-mouse or goat anti-rabbit $\mathrm{IgG}$ for $20 \mathrm{~min}$ at $37^{\circ} \mathrm{C}$. The sections were then incubated with 3,3'-diaminobenzidine (DAB) chromogen for 5-10 min at room temperature and washed with distilled water. Finally, the slides were washed for $5 \mathrm{~min}$ in running tap water and counterstained with Harris hematoxylin.

Immunohistochemical analysis. A positive value was recorded for HIF-1 $\alpha$ and Ki67 immunoreactivity when nuclear staining was observed. Using cytoplasm staining, LC3 and Atg4B were found to be positive. Semi-quantitative expression levels were determined by assessing the percentage and intensity of the stained tumor cells. The percentage of positive cells was rated per HPF using a magnification of $\times 200$ as follows: cases with $<1 \%$ positive cells were rated as zero, $1-25 \%$ positive cells as 1 point, $26-50 \%$ positive cells as 2 points and $>51 \%$ positive cells as 3 points. The staining intensity was rated as: 1 point for weak, 2 points for moderate and 3 points for strong intensity. The points for staining intensity and the percentage of positive cells were added together. The specimens were then classified into three groups according to their overall score: negative expression for $0-1$ point, weak expression for 2-4 points and a strong expression for 5-6 points.

Total RNA isolation and real-time PCR. Primers were designed and synthesized by Takara Biotechnology Co., Ltd. Real-time PCR analysis was carried out to determine the mRNA expression of LC3 and Atg4B using the Gene AMP PCR system 7500 sequence detector. A separate PCR assay for the mRNA of the $\beta$-actin housekeeping gene was performed to verify general mRNA integrity. Total RNA was extracted from 30 melanoma tumor samples frozen at $-80^{\circ} \mathrm{C}$ using TRIzol reagent. The optical density (OD) 260/280 ratio for all 70 samples was 1.9-2.0. RNA was then converted into cDNA using Taq Man reverse transcriptase reagents (Applied Biosystems). cDNA was used as the template that was amplified in a $25-\mathrm{ml}$ reaction mixture using the following conditions: denaturation at $94^{\circ} \mathrm{C}$ for $5 \mathrm{~min}$, then 35 cycles of $94^{\circ} \mathrm{C}$ for 

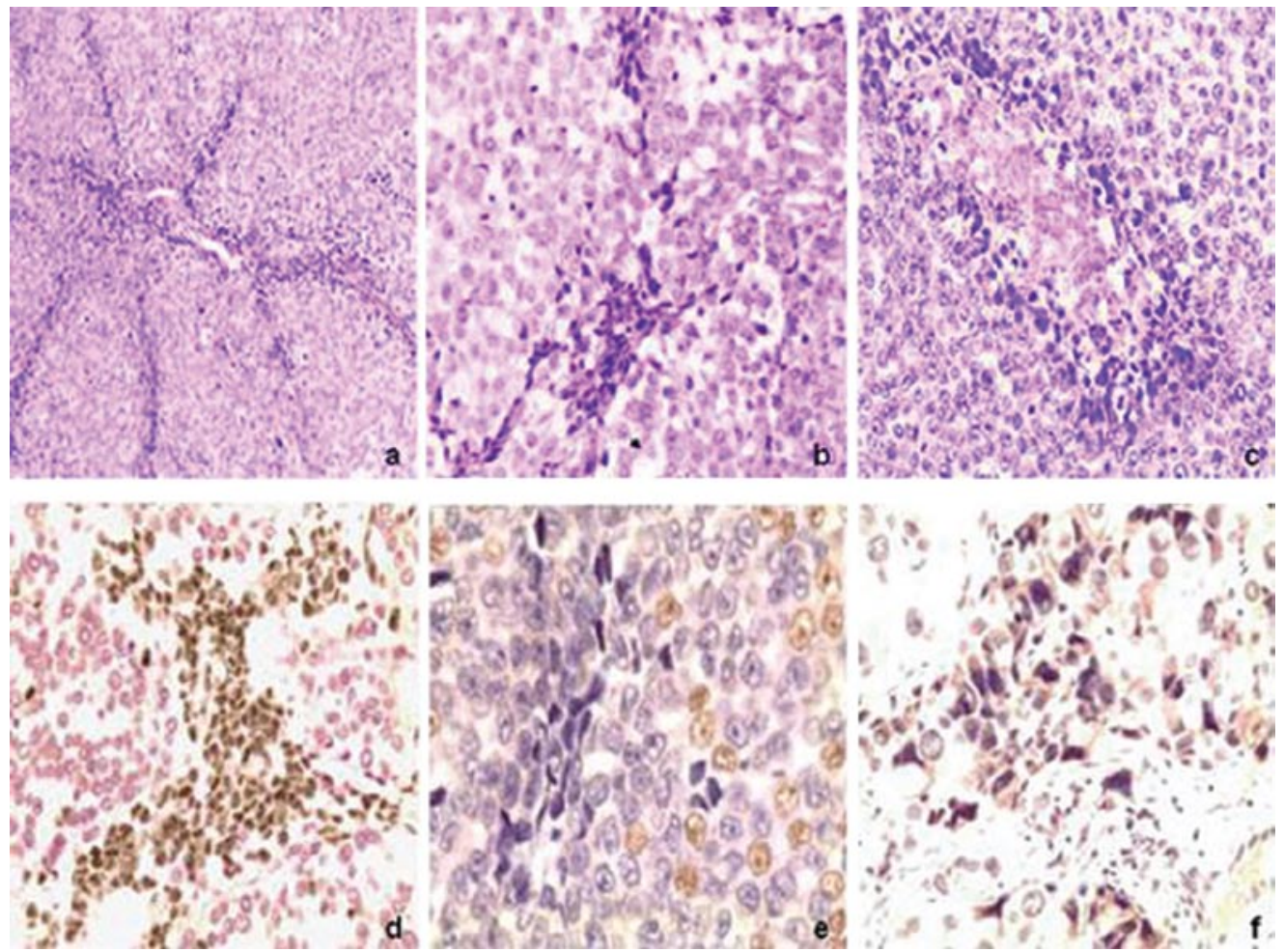

Figure 1. (a) and (b) Cells that underwent LPPCN are darkly stained and distributed in patterns of lines and/or networks among the environmental tumor cells. These networks are accompanied with neovasculogenesis networks. Inflammation cells are absent in the environmental district. (c) In the environment of LPPCN, lamellae of conventional necrosis are present, and the neovasculogenesis networks are absent. (d) Immunohistochemical staining for LC3 shows that cells that underwent LPPCN are positive in the cytoplasm. (e) Immunohistochemical staining for Ki67 shows that cells that underwent LPPCN are negative, but cells around them are positive. (f) Immunohistochemical staining for Atg4B shows that cells that underwent LPPCN are positive in the cytoplasm.

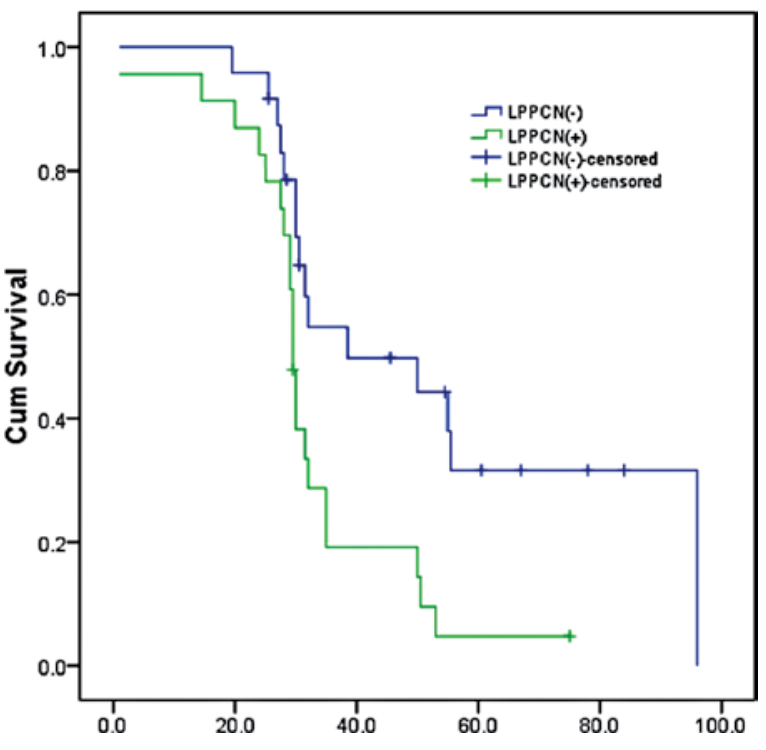

Figure 2. Kaplan-Meier survival analysis showed that the survival time for patients with the presence of LPPCN is significantly shorter than that for patients without LPPCN.

$30 \mathrm{sec}$, the optimal annealing temperature for $45 \mathrm{sec}$ and $55^{\circ} \mathrm{C}$ for $40 \mathrm{sec}$. The primer sequences used for the matrix metalloproteinase LC3 (Accession no.: AF087871.1) detection were 5'-GGCGCTTACAGCTCAATGCTAAT-3' (sense) and 5'-AAT TTCATCCCGAACGTCTCCTG-3' (antisense). The primer sequences used for Atg4B (Accession no.: NM_013325.4) detection were 5'-ATGGAGGAAATCAGAAGGTTGT-3' (sense) and 5'-TCGTTGATGTCCGTGAGCC-3' (antisense). The primers used to amplify $\beta$-actin were 5'-ATCCGTAAAGAC CTCTATGCCAAC-3' (sense) and 5'-ATGGAGCCACCGAT CCACA-3' (antisense). The resultant products of LC3, Atg4B and $\beta$-actin amplification were 163,185 and 174 base pairs, respectively. The $\mathrm{CT}$ value (the cycle number at which the fluorescence crosses the threshold) was determined and the formula $2^{-\Delta \Delta \mathrm{Ct}}$ was used to determine the relative quantity of the amplified fragment. Each sample was tested in triplicate and the mean value was used.

Statistical analysis. Statistical analysis was performed in this study using SPSS 13.0. Pearson's $\chi^{2}$ test. Bivariate correlations were used to determine the different parameters associated with the distribution of LPPCN. A t-test was used to analyze the mRNA expression of LC3 and Atg4B. The Kaplan-Meier method and the log-rank test were employed to evaluate survival analysis where $\mathrm{p}<0.05$ was considered to be significant.

\section{Results}

Morphological characteristics of linearly patterned programmed cell necrosis. In the H\&E-stained sections of tumor tissues of melanoma, cells that underwent LPPCN were characterized by darker staining than that of the environmental cells, loss of cell-cell adhesion, concentrated cytoplasm, cellular nucleus adopting a different pattern or heteromorphies (e.g., triangulum, polygon, fusiform and 
Table I. Correlations between LPPCN and clinical and histopathological parameters in melanoma.

\begin{tabular}{cccccc}
\hline Parameter & Total & & LPPCN & p-value \\
& no. & - & + & Positive & \\
& & & & rate, $\%$ & \\
& & & & &
\end{tabular}

\section{Gender}

Male

Female$$
43
$$

43

23

Age

$\leq 55$
$<55$

27

2320

46.5

66.7

0.253

0.099

Tumor location

Trunk
Extremities

38
32

$\begin{array}{ll}15 & 23 \\ 17 & 15\end{array}$

60.5

46.9

LN metastasis

$$
\text { Absent }
$$

Present

$\begin{array}{lll}43 & 16 & 27\end{array}$

62.8

40.7

DM

Absent
Present
AJCC stage
I+II
III+IV

Tumor thickness

$$
\leq 17 \mathrm{~mm}
$$

$>17 \mathrm{~mm}$

27

16

11

0.071

0.150

Histology

$\mathrm{A}+\mathrm{N}+\mathrm{Un}$
$\mathrm{SSM}$

Melanin

$$
\begin{aligned}
& \text { Absent } \\
& \text { Present }
\end{aligned}
$$

$\begin{array}{lll}35 & 13 & 22\end{array}$

$35 \quad 19 \quad 16$

0.224

48.9

64.0

55.6

$\begin{array}{llll}27 & 12 & 15 & 55.6 \\ 43 & 20 & 23 & 53.5\end{array}$

Nucleolus

$\begin{array}{lrrrr}\text { Absent } & 36 & 19 & 17 & 47.2 \\ \text { Present } & 34 & 13 & 21 & 61.8 \\ \text { CP } & & & & \\ \text { Spindle } & 28 & 10 & 18 & 64.3 \\ \text { Epithelium } & 27 & 16 & 11 & 40.7 \\ \text { Diphase } & 15 & 6 & 9 & 60.0 \\ \text { T-IL } & & & & \\ \text { Absent } & 34 & 14 & 20 & 58.8 \\ \text { Present } & 36 & 18 & 18 & 50.0\end{array}$

Conventional necrosis

$\begin{array}{lrrrr}\text { Absent } & 32 & 25 & 7 & 21.9 \\ \text { Present } & 38 & 7 & 31 & 81.6\end{array}$

LN, lymph node; DM, distant metastasis; AJCC, American Joint Committee on Cancer Staging; SSM, superficial spreading melanoma; CP, cellular phenotype; T-IL, tumor-infiltrating lymphocytes; A+ N+Un: ALM+ NM+Untyped.

0.866

0.018

0.025

0.222

0.000
Table II. Correlations between LPPCN and immunohistochemical parameters in melanoma.

\begin{tabular}{lcccc}
\hline Protein & LPPCN $(\mathrm{p})$ & LC3 $(\mathrm{p})$ & $\operatorname{Atg} 4 \mathrm{~B}(\mathrm{p})$ & $\mathrm{Ki} 67(\mathrm{p})$ \\
\hline LC3 & $0.01^{\mathrm{a}}$ & & & \\
Atg4B & $0.01^{\mathrm{a}}$ & $0.010^{\mathrm{a}}$ & & \\
Ki67 & $0.000^{\mathrm{a}}$ & 0.200 & $0.015^{\mathrm{a}}$ & \\
Hif-1 $\alpha$ & $0.000^{\mathrm{a}}$ & $0.004^{\mathrm{a}}$ & $0.000^{\mathrm{a}}$ & $0.000^{\mathrm{a}}$ \\
\hline
\end{tabular}

p, p-value; asignificance.

Table III. Expression of LC3 and Atg4B mRNA in melanoma.

\begin{tabular}{lcccr}
\hline Genes & Group & $\overline{\mathrm{x}} \pm \mathrm{SD}$ & t-test & p-value \\
\hline Atg4B & & & 2.63 & 0.020 \\
& LPPCN $^{+}$ & $3.605 \pm 0.881$ & & \\
& LPPCN $^{-}$ & $2.372 \pm 0.950$ & & \\
LC3 & & & 6.204 & 0.000 \\
& LPPCN $^{+}$ & $5.793 \pm 1.467$ & & \\
& LPPCN $^{-}$ & $2.124 \pm 0.963$ & & \\
\hline
\end{tabular}

deformed pattern) and bolus-shaped and homogeneous chromatin. The cells were distributed in patterns of lines and/or networks among the environmental tumor cells. The LPPCN lines and/or networks observed were occasionally accompanied with neovasculogenic networks or conventional necrosis. Inflammation cells were absent in the environmental district of LPPCN (Fig. 1 a-f). The morphological changes of LPPCN are similar to neither apoptosis, which is typically characterized by apoptotic bodies, nor conventional necrosis, which is typically characterized by electron-lucent cytoplasm, swelling of cellular organelles, loss of plasma membrane integrity and the inflammatory reaction presents.

Correlations between linearly patterned programmed cell necrosis and clinical and histopathological parameters. A total of $54.29 \%(38 / 70)$ of the specimens observed exhibited the LPPCN phenomenon. The appearance of LPPCN has a close positive correlation with the following parameters in melanoma: tumor thickness $(\mathrm{p}=0.016)$, histological subtype $(\mathrm{p}=0.029)$ and conventional necrosis $(\mathrm{p}=0.000)$, but has a negative correlation with melanin $(\mathrm{p}=0.001)$. The presence of LPPCN did not show any association with gender, age, lymph node invasion, AJCC stage, cellular phenotype, tumorinfiltrating lymphocytes or nucleolus $(\mathrm{p}>0.05)$. Table I shows the clinical and histopathological characteristics involved in LPPCN.

Correlation between LPPCN and immunohistochemical activities. Immunohistochemical staining for Ki67 showed that cells that underwent LPPCN were negative, while tumor cells that were distant to LPPCN were positive. For HIF-1 $\alpha$, cells that underwent LPPCN were found to be positive. The positive rates of $\mathrm{Ki} 67$ and HIF- $1 \alpha$ in the group where LPPCN 
was present were higher than those in the group without LPPCN ( $<<0.05)$, a finding that is the same as the results of LC3 and Atg4B. Correlation analysis showed that the expression of both LC3 and Atg4B are closely correlated ( $\mathrm{p}=0.010)$. Data further showed that the presence of LPPCN is related with the Ki67 index $(p=0.000), \operatorname{LC} 3(p=0.01)$ and Atg4B $(\mathrm{p}=0.01)$ (Table II).

Expression of LC3 and Atg $4 B$ mRNA. Real-time PCR was performed to detect LC3 and Atg4B mRNA expression in the LPPCN-positive and -negative groups in melanoma. The expression of LC3 and Atg4B mRNA in the LPPCN-positive group was higher than that of the LPPCN-negative group. The difference was significant $(\mathrm{p}=0.000$ and $\mathrm{p}=0.02)$, respectively (Table III).

Survival analysis. Kaplan-Meier survival analysis showed that the survival time for patients with the presence of LPPCN was significantly shorter than that for patients without LPPCN. The average survival time of cases with and without the presence of LPPCN was 28.870 \pm 2.567 and 53.255 \pm 6.809 months, respectively $(\mathrm{p}<0.05)$. In addition, an analysis of the results showed that parameters such as tumor thickness, histological subtype, melanin, conventional necrosis, cellular phenotype, tumor-infiltrating lymphocytes, lymph node invasion and Ki67 index had a strong influence on the survival of patients with melanoma $(\mathrm{p}<0.05)$ (Fig. 2).

\section{Discussion}

No efficient chemotherapy for melanoma currently exists $(16,17)$. Even in low-incidence countries such as China, the status remains a major concern. A novel phenomenon of cell death known as LPPCN is noted in melanoma (3). This study, for the first time, carefully observed and described tumor cell subpopulations that underwent LPPCN. It confirmed that the LPPCN phenomenon is a novel but common characteristic in more aggressive melanoma. Morphological characteristics associated with LPPCN have been sparsely described by pathologists thus far. Subsequently, we carefully evaluated the distribution and quantification of LPPCN in human melanoma. Cells that underwent LPPCN were morphologically characterized by loss of cell-cell adhesion, concentrated cytoplasm and a cellular nucleus stained darkly in H\&E-stained sections. The cells were distributed in patterns of lines and/or networks within the environmental tumor cells. The cell clusters and/or networks were always accompanied with neovasculogenesis networks or conventional necrosis, but inflammatory cells were absent in the environmental district of LPPCN.

Based on morphological observations of LPPCN, immunohistochemical experiments were performed to determine the formation mechanisms of LPPCN. Findings show that LPPCN is a special type of PCD that can be activated by many factors, such as tissue hypoxia, drugs and toxins (18). Additionally, cells underwent programmed necrosis regulated by the tumor cells themselves under tumor microenvironmental conditions $(19,20)$. In this study, cells that underwent LPPCN tested negative for Ki67, but positive for HIF-1 $\alpha$ by immunohistochemical staining. This phenomenon suggests that the formation of dying cell networks through LPPCN may be initiated in the local hypoxic microenvironment under which HIF-1 $\alpha$, which mainly mediates the hypoxic response, was up-regulated, and that these cells underwent programmed necrosis (3). Notably, the distribution and morphological changes of LPPCN are typically different from apoptosis and conventional necrosis. Cells that underwent LPPCN formed clusters, lines or networks. By contrast, apoptosis reflects scattered cells in randomly distributed tumor tissues. Necrosis usually occurs within the lamellar tracts of contiguous cells in H\&E-stained sections (21). Furthermore, morphologically, LPPCN cells showed loss of cell-cell adhesion, concentrated cytoplasm, pyknosis, karyorrhexis and karyolysis; characteristics that are different from the apoptotic bodies and DNA fragmentation identified. By contrast, necrosis was characterized by a pyknotic nucleus, cytoplasmic swelling, progressive disintegration of the cytoplasmic membranes and inflammation cell reaction. Additionally, apoptotic cell death required energy in the form of ATP. We were therefore able to infer that, under hypoxic microenvironments which were unable to supply sufficient energy to cell death, LPPCN most likely avoided consuming energy to conform to the hypoxic microenvironment.

The hypothesis that cells perish by mechanisms other than apoptosis has been gaining momentum (6). Technological advances have aided in defining the role and mechanism of programmed necrosis, as well as the role of autophagy in cell survival and disintegration (8). Autophagy is also considered to be a potential mechanism for non-apoptotic and/or nonnecrosis cell death despite evidence from a number of species that it is a survival strategy in times of stress (6). In this study, immunohistochemical results show that the overexpression of LC3 and Atg4B at the protein and mRNA levels in the LPPCNpositive group as opposed to the LPPCN-negative group in melanoma, as well as the expression of LC3 and Atg4B, are closely correlated. Thus, we proposed that autophagy plays a crucial role in LPPCN. Endo G was thought to play a role in the process of LPPCN in a study by Zhang et al (3). We speculated whether apoptotic and autophagic mechanisms contribute to LPPCN as certain findings indicated in curcumin-induced K562 cell death (22). Numerous consecutive experiments are required in order to verify this novel phenomenon.

According to Zong et al (5), chemicals such as $\mathrm{Ca}^{2+}$ chelators, ROS scavengers, PARP inhibitors, calpain inhibitors and cathepsin inhibitors have been shown to inhibit programmed necrotic cell death. Studies using gene knockout models in $C$. elegans and mice have also been useful in showing that programmed necrotic cell death can be prevented (5). Determining the regulatory mechanisms for LPPCN is crucial. A better understanding of the regulating factors involved in programmed necrosis should allow future studies to evaluate the role of LPPCN in solid tumors more thoroughly.

In 1971, Folkman (23) hypothesized that tumor expansion and metastasis are dependent upon angiogenesis. Angiogenesis is a well-orchestrated sequence of events that involves endothelial cell migration, proliferation, degradation of tissue, new capillary vessel (sprout) and loop formation (anastomosis), as well as the blood flow associated with the nascent network (24). However, a crucial point that remains to be elucidated is how a solid tumor with compact cell mass and a high IFP (25) interior is able to provide spacial functions 
to facilitate endothelial cell migration and proliferation. The findings regarding LPPCN may clarify this concern during angiogenesis.

Prior to vasularization, various solid tumors are found in animals that live as tiny spheroids or ellipsoids of a few millimeters in diameter, and which are dependent on simple diffusion for absorption of nutrients and release of catabolites (23). Tumors become dormant at a diameter of only a few millimeters due to the absence of blood vessels. However, once vascularized, these tumors are released from the dormant phase and begin exponential growth (26). In a microenvironment without adequate oxygen and blood supply, certain tumor cells are likely to be controlled by regulators of PCD, such as Endo G, LC3 and Atg4B. LPPCN is then initiated and results in a channel-shaped, vessel-like empty space left by the dead cells. Subsequently, blood vascular networks can be constructed via the proliferation of the endothelial cells migrating towards the middle of the tumor following the networks left by LPPCN. Without endothelial cell ingression into the tumor that would allow vessel formation, the tumor would undergo conventional necrosis. Therefore, we speculated that LPPCN was an early stage event in tumoral neovascularization.

This study provides a comprehensive investigation of the associations between the occurrence of LPPCN and various clinicopathological parameters in melanoma. The results indicated that LPPCN has a close correlation with various clinicopathological parameters in melanoma, including tumor thickness, histological subtype, conventional necrosis and melanin. In this study, the Kaplan-Meier method and log-rank test data showed that the survival time of patients with LPPCN-positive tumors is shorter than that of patients without LPPCN. This is similar to the result of Zhang et al (3). Therefore, we assume that LPPCN correlates with a poor patient outcome.

In conclusion, the data from our experiment confirmed that LPPCN extensively exists in melanoma. The cellular subpopulations that underwent LPPCN were previously ignored by pathologists. LPPCN may be a special type of PCD, and an autophagic mechanism may contribute to it. LPPCN has a close correlation with certain histopothalogical characteristics and signifies a poor prognosis for patients with melanoma. We speculated that LPPCN was an early stage event in tumoral neovascularization. Although the mechanism involved in LPPCN remains unclear and subsequent investigations are necessary to verify this novel phenomenon, LPPCN can be considered a novel target in the process of antiangiogenesis treatment, leading to an evolutionary strategy for melanoma in the future.

\section{Acknowledgements}

This study was supported by a grant from the National Nature Science Foundation of China (Grant No. 30770828).

\section{References}

1. McKinnon JG, Yu XQ, McCarthy WH, et al: Prognosis for patients with thin cutaneous melanoma: long-term survival data from New South Wales Central Cancer Registry and the Sydney Melanoma Unit. Cancer 98: 1223-1231, 2003.

2. American Cancer Society: Cancer Facts And Figures. GA: American Cancer Society; Atlanta, 2008.

3. Zhang SW, Li M, Zhang DF, et al: Hypoxia influences linearly patterned programed cell necrosis and tumor blood supply patterns formation in melanoma. Lab Invest 89: 575-586, 2009.

4. Assuncao Guimaraes C and Linden R: Programmed cell deaths. Apoptosis and alternative deathstyles. Eur J Biochem 271: 1638-1650, 2004.

5. Zong WX and Thompson CB: Necrotic death as a cell fate. Genes Dev 20: 1-15, 2006.

6. Yuan J, Lipinski M and Degterev A: Diversity in the mechanisms of neuronal cell death. Neuron 40: 401-413, 2003.

7. Zong WX, Ditsworth D, Bauer DE, et al: Alkylating DNA damage stimulates a regulated form of necrotic cell death. Genes Dev 18: 1272-1282, 2004.

8. Edinger AL and Thompson CB: Death by design: apoptosis, necrosis and autophagy. Curr Opin Cell Biol 16: 663-669, 2004.

9. Kitanaka $\mathrm{C}$ and Kuchino Y: Caspase-independent programmed cell death with necrotic morphology. Cell Death Differ 6: 508-515, 1999.

10. Satoo K, Noda NN, Kumeta H, et al: The structure of Atg4B-LC3 complex reveals the mechanism of LC3 processing and delipidation during autophagy. EMBO J 28: 1341-1350, 2009.

11. Philip EL, Burg G, David W and Sarasain A (eds.): World Health Organization Classification of Tumours Pathology and Genetics of Skin Tumours. IARC Press, Lyon, 2006.

12. Bursch W, Ellinger A, Gerner C, Fröhwein U and SchulteHermann R: Programmed cell death (PCD). Apoptosis, autophagic PCD, or others? Ann NY Acad Sci 926: 1-12, 2000.

13. Tolkovsky AM: Mitochondrial disappearance from cells: a clue to the role of autophagy in programmed cell death and disease? Biochimie 84: 233-240, 2002.

14. Larsen KE and Sulzer D: Autophagy in neurons: a review. Histol Histopathol 17: 897-908, 2002.

15. Cuervo AM: Autophagy: in sickness and in health. Trends Cell Biol 14: 70-77, 2004

16. Osterlind A: Epidemiology on malignant melanoma in Europe. Acta Oncol 31: 903-908, 1992.

17. Karim-Kos HE, de Vries E, Soerjomataram I, Lemmens V, Siesling S and Coebergh JW: Recent trends of cancer in Europe: a combined approach of incidence, survival and mortality for 17 cancer sites since the 1990s. Eur J Cancer 44: 1345-1389, 2008.

18. Yakolev AG and Faden AI: Mechanisms of neural cell death: implications for development of neuroprotective treatment strategies. NeuroRx 1: 5-16, 2004.

19. Proskuryakov SY, Gabai VL and Konoplyannikov AG: Necrosis is an active and controlled form of programmed cell death. Biochemistry (Mosc) 67: 387-408, 2002.

20. Lugovskoy AA, Degterev AI, Fahmy AF, et al: A novel approach for characterizing protein ligand complexes: molecular basis for specificity of small-molecule Bcl-2 inhibitors. J Am Chem Soc 124: 1234-1240, 2002.

21. Wyllie AH, Kerr JF and Currie AR: Cell death: the significance of apoptosis. Int Rev Cytol 68: 251-306, 1980.

22. Jia YL, Li J, Qin ZH and Liang ZQ: Autophagic and apoptotic mechanisms of curcumin-induced death in K562 cells. J Asian Nat Prod Res 11: 918-928, 2009.

23. Folkman J: Tumor angiogenesis: therapeutic implications. N Engl J Med 285: 1182-1186, 1971.

24. Carmeliet P: Mechanisms of angiogenesis and arteriogenesis. Nat Med 6: 389-395, 2000.

25. Tannock IF and Hayashi S: The proliferation of capillary endothelial cells. Cancer Res 32: 77-82, 1972.

26. Folkman J and Hochberg M: Self-regulation of growth in dimensions. J Exp Med 138:745-753, 1973. 